\title{
Studies on Frying Quality of Virgin Coconut Oil and Shortening Blends
}

\author{
MUHAMMAD NOR OMAR, M H NOR-HAZWANI, \\ MUHD N. M. NAZREEN and AHMAD MUZAMMIL ZUBERDI
}

Kulliyyah of Science, International Islamic University Malaysia, Indera Mahkota, 25200 Kuantan Pahang Malaysia.

http://dx.doi.org/10.13005/ojc/300344

(Received: July 30, 2014; Accepted: August 31, 2014)

\begin{abstract}
The frying performances of palm solid shortening (PS) and virgin coconut oil (VCO) blends were evaluated. The fresh chickens were fried in a blended frying medium using an open fryer for 8 hours per day intermittently at 30 min interval for five consecutive days. Three types of oils were used; i.e. palm shortening without blending (PS), a blend of VCO: PS (10:90), and a blend of VCO: PS (20:80). The frying oils were collected periodically and their fatty acid composition (FAC), free fatty acid (FFA) content, total polar compound (TPC) and total polymer material (TPM) were determined. In addition, the fried chicken was subjected to the analyses of oil absorption and sensory evaluation. The results showed that the frying performance of the VCO:PS (20:80) blend was better than VCO:PS (10:90) blend and PS in terms of TPM and TPC contents. The VCO:PS (20:80) gave TPC and TPM values of 17.4 and $1.40 \%$ respectively, the blend of VCO:PS (10:90) gave values of TPC and TPM of 18.7 and $2.20 \%$ respectively, whilst PS with TPC of and TPM of 20.5 and $3.02 \%$ respectively. Meanwhile, the oil absorption patterns showed that the fried chicken in VCO: PS (10:90) blend absorbed less oil compared to chickens fried in PS and VCO:PS (20:80) blend. The oil uptake in chickens fried in VCO:PS (10:90) blend was 1.75\%, while in PS and VCO:PS (20:80) blend were 2.82 and $2.57 \%$ respectively.In term of sensory evaluation, the addition of virgin coconut oil (VCO) to palm based solid shortening (PS) showed significant differences $(p<0.05)$ on crispiness, taste and overall scores on the $1^{\text {st }}, 3^{\text {rd }}$ and $5^{\text {th }}$ day of frying. Although there were no significant difference on oiliness on the $1^{\text {st }}$ and $3^{\text {rd }}$ day, there was a significant difference on the $5^{\text {th }}$ day of frying. In conclusion, the addition of a small proportion of VCO in PS would improve the frying performance of oil as well as sensory scores of the fried products.
\end{abstract}

Key words: Frying performance, oil absorption, palm shortening (PS), virgin coconut oil (VCO).

\section{INTRODUCTION}

Fat contents in fried products become a great concern among consumers as taking an excess fat may contribute to overweight and obesity. As much 300 million people in the world population, as well as $64 \%$ of the United States adult population are considered either overweight or obese $e^{1,2,3}$ and 
this percentage has increased over the last four decades. It has been concluded that, a large number of medical dysfunctions have been associated with obesity/overweight including osteoarthritis, diabetes, cancer, cardiovascular and liver diseases ${ }^{4,5}$.

Nowadays, many fast food outlets are using vegetable frying medium to produce fried products, such as fried chicken, burgers and potato chips. All these products absorb oils between $6-15 \%$. Potato chips will absorb oil up to $14 \%$ when frying in palm olein 6 . A similar pattern on oil uptake is also observed when using different frying medium, including soybean oil, canola oil, olive oil and sunflower oil.

Many techniques and treatments are used in order to reduce oil uptake in fried products and improve the frying performance of the oil. One of the common techniques is by using a shortening and its blends. It has been found that, potato chips absorbed ca. $4.8 \%$ when frying in palm solid shortening (PS) ${ }^{7}$, and further reduced to $4.3 \%$ when the shortening is blended with palm olein ${ }^{8}$. In terms of the total polar content (TPC) and the total polymeric material (TPM), the values obtained from palm solid shortening (PS) are lower than from palm olein (POo), and these values are further improved when using palm shortening-olein blends ${ }^{7,8}$. In addition, PS also contains no trans fatty acids hence improves the human health ${ }^{7,9}$.

The virgin coconut oil (VCO), which has been manufactured through fermentation and centrifugation technique has a lot of beneficial effects since it contains vitamin $\mathrm{E}$ and polyphenol ${ }^{10}$. The effects include lowering the lipid levels in serum and protecting low-density lipoprotein against oxidative stress ${ }^{11}$. In frying, the total oxidation value and polar compound in VCO is lower than in palm olein due to the presence of polyphenol compounds ${ }^{12}$.

The main objective of the present study is to evaluate the frying performance of palm solid shortening (PS) against VCO:PS blends using an open batch fryer. Besides that, assessment of the sensory qualities and oil uptake of fried products will also be carried out.

\section{EXPERIMENTAL}

\section{Sample preparation}

The fresh chickens were obtained from local market in December 2013. After chopping into small pieces, the chickens were washed and rinsed prior to frying. The palm shortening was obtained from local outlets while virgin coconut oil (VCO) was prepared in-house according to method previously reported ${ }^{13}$.

\section{Frying experiments}

The experiment was carried out using a One Well electric open fryer (IEF 171, one pot table top, Kualal Lumpur, Malaysia) according to the method previously reported ${ }^{7}$. The palm solid shortening (PS) and virgin coconut oil (VCO) blends were used to fry fresh chickens for $10 \mathrm{~min}$ at $180+2^{\circ} \mathrm{C}$. An intermittent frying experiment was carried out for 8 hours per day over five consecutive days. Eight batches of chickens, with every batch weighing approximately $350 \mathrm{~g}$, were fried daily with an interval of 60 minutes between batches. For the analysis of the physicochemical parameters, an oil sample of $100 \mathrm{ml}$ was taken at the end of each day of frying. The samples were cooled, purged with nitrogen and kept at $-18^{\circ} \mathrm{C}$ before analyses. The fried chicken samples were collected each day at the end of frying process and were kept at ambient temperature for sensory evaluation and oil uptake analysis.

\section{Physicochemical Analysis of Fats Oil uptake analysis}

Total oil content of fried chickens was determined by Soxhlet extraction according to reported method ${ }^{7}$. The oil was extracted using a Soxtec $^{\mathrm{TM}} 2050$ Auto Fat Extraction System (Foss Analytical AB, Hoganas, Sweden). Two (2) gram of fried chicken sample was extracted for $2 \mathrm{~h}$ using $\mathrm{n}$-hexane as a solvent.

\section{Fatty Acid Composition Analysis by GC}

The oil sample was methylated using a boron trifluoridemethanolic sodium hydroxide solution. The oil was analysed via on-column GC technique using Agilent 6890N gas chromatograph (Agilent, Avondale, USA) equipped with a flame ionization detector (FID). An HP-5 non-polar capillary column (50 m x $0.12 \times 0.5 \mathrm{~mm}$, SGE, Australia) was used and the temperature was initially kept at $50^{\circ} \mathrm{C}$ for $2 \mathrm{~min}$ and the programmed at $5^{\circ} \mathrm{C}$ per min to 
$250^{\circ} \mathrm{C}$. The injector and detector temperature were $220^{\circ}$ and $250^{\circ} \mathrm{C}$ respectively and $\mathrm{He}$ gas was used as carrier gas with a flow rate of $1.0 \mathrm{~mL}$ per min.

\section{HPLC analysis of Total Polar Content and Total Polymeric Material}

The total polar content (TPC) and the total polymeric material (TPM) were determined by HPLC technique according to methods previously reported ${ }^{7,14,15}$. HPLC analysis was carried out using a Waters HPLC system (Waters Associates, Milford, MA) equipped with a Rheodyn 7725 injector (10 $\mathrm{ml}$ sample loop), Waters 510 HPLC pump, $100^{\circ} \mathrm{A}$ Ultrastyle column and a refractive index detector.

\section{Free Fatty Acid (FFA)}

The free fatty acid (FFA) was determined as palmitic acid according to the MPOB Test Method ${ }^{16}$, which has been adopted from AOCS ${ }^{17}$.

\section{Sensory Evaluation}

For sensory evaluation, sufficient samples of freshly fried chickens were taken on the day 1 , 3 and 5 of frying experiment and then served to trained panelists within $10 \mathrm{~min}$ after taken out of the fryer. Panelists were requested to evaluate the organoleptic attributes, i.e. colour, odour, oiliness, crispiness, taste and overall acceptance) of fried chickens. A nine-point hedonic scoring scale was used by the panel members to judge their perception, where 9 = extremely good and $1=$ extremely bad. The sensory scores will be analyzed statistically using SPSS software (version 18.0 for Windows; SPSS Inc., Chicago, IL, USA).

\section{RESULTS AND DISCUSSION}

\section{Oil uptake analysis}

Figure 1 shows that the percentage of oil uptake/absorption by the chickens fried in three types of frying oils. By subtracting the initial oil content $(0.12 \%)$ in the raw chicken, it was found that the oil uptake was the lowest in the chicken fried using VCO:PS (10:90) blend. During frying process, it was decreased from $3.39 \%$ to $1.75 \%$ whereas in PS, it was decreased from $3.15 \%$ to $2.82 \%$. Meanwhile, the oil absorption of fried chickens in VCO: PS (20:80) was increased from 2.08 to $2.57 \%$. The decreasing in oil uptake might be due to the presence of linolenic acid. As reported by Kita and Lisinska ${ }^{18}$, the fat absorption in fried products will be low when the amount of unsaturated fatty acid, i.e. linoleic acid in the frying medium is low. Analysis of fatty acid composition (FAC) showed that linoleic acid content of VCO: PS (10:90) blend (3.8\%) was the lowest compared to its content VCO:PS (20:80) blend (3.9\%) and PS (4.3\%).

\section{Analysis of fatty acid composition (FAC)}

Table 1 shows the fatty acid composition of PS and VCO:PS blends. The VCO oil indicated that very high proportion of saturated fatty acid $(94.8 \%)$ while the unsaturated fatty acid was only $5.2 \%$. Apart from that, lauric acid in VCO constituted $51.2 \%$ while linoleic acid is $0.47 \%$. When blending with PS, lauric acid content was the highest in VCO: PS $(20: 80)$ blend $(12.58 \%)$ compared to $4.24 \%$ in VCO: PS (10:90) blend and $0.07 \%$ in PS (Table 2). Consequently, before frying, VCO: PS (20:80) blend contained the highest proportion of saturated fatty acids $(69.3 \%)$ as compared to VCO: PS (10:90)

Table 1: Percentages of saturated and unsaturated fatty acid of virgin coconut oil

\begin{tabular}{lc}
\hline Types of fatty acid & Percentage (\%) \\
\hline Saturated fatty acid & 94.8 \\
Unsaturated fatty acid & 5.20 \\
Lauric acid & 51.2 \\
Linoleic acid & 0.47 \\
\hline
\end{tabular}

Table 2. Fatty acid composition (FAC) of blended shortenings

\begin{tabular}{lcccccccc}
\hline Types of oil & C8:0 & C10:0 & C12:0 & C14:0 & C16:0 & C18:2 & C18:1 & C18:0 \\
\hline Control oil (PS) & 0 & 0 & 0.07 & 1.06 & 52.96 & 6.33 & 34.12 & 5.47 \\
VCO/PS (10:90) & 0.42 & 0.47 & 4.24 & 2.62 & 43.01 & 2.82 & 39.89 & 6.53 \\
VCO/PS (20:80) & 2.19 & 1.69 & 12.58 & 5.23 & 43.10 & 5.13 & 25.53 & 4.55 \\
\hline
\end{tabular}


(57.3\%) and PS (59.6\%) (Figure 2). After the $5^{\text {th }}$ day of frying, VCO: PS (20:80) blend still contained a higher amount of saturated fatty acids $(67.3 \%)$ than VCO: PS (10:90) blend (64.7\%) and PS (62.3\%). In term of linoleic acid content, it was showed that VCO: PS (10:90) blend has the highest linoleic acid (2.82\%) compared to VCO: PS (20:80) blend (5.13\%) and PS (6.33\%). On the $1^{\text {st }}$ day of frying, linoleic acid decreased to $5.04 \%$ in PS and $3.92 \%$ in VCO: PS (20:80) blend while increased to $5.93 \%$ in VCO: PS (10:90) blend (Figure 3). After frying for 5 days, linoleic acid was decreased to $4.31 \%$ in PS and $3.81 \%$ in VCO: PS (20:80) blend while in VCO: PS (10:90) blend, it was increased to $3.82 \%$. The lauric acid content was also changed significantly. On the $5^{\text {th }}$ day of frying, VCO: PS (20:80) blend still consisted the highest content of lauric acid which the value of $8.9 \%$ compared to VCO: PS (10:90) blend (4.3\%) and PS (0.13\%) (Figure 4). Lauric acid is a medium

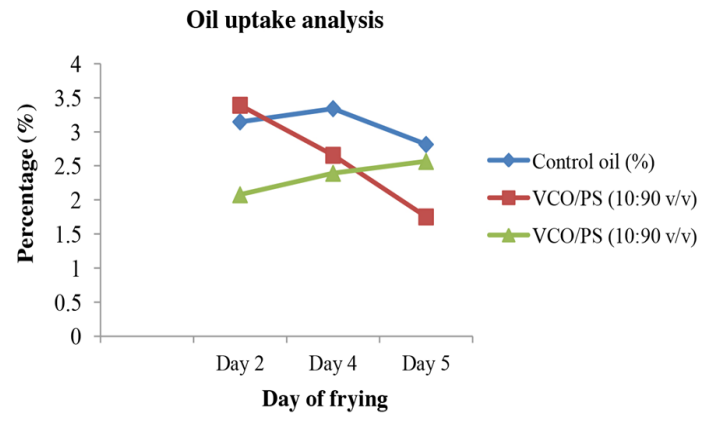

Fig. 1: Oil uptake in fried chickens during frying experiment using blended shortening

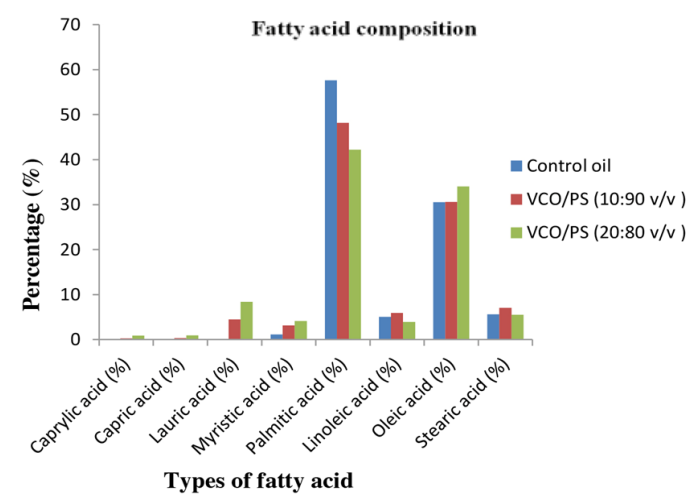

Fig. 3: Fatty acid composition (FAC) in blended shortenings after the $1^{\text {st }}$ day of frying chain fatty acid which found abundant in coconut oil and VCO that acts as antioxidant properties that may capable to increase the stability of oil during frying ${ }^{20}$. The addition of virgin coconut oil in vegetable oil believed to reduce the oil degradation during frying as reported by Ghazali et al ${ }^{12}$, virgin coconut oil capable to increase the stability of oil during frying because the mode of virgin coconut oil extraction retains more biologically active component such as vitamin $\mathrm{E}$ and polyphenol compared to coconut oil.

\section{Free fatty acid content}

In this study, the free fatty acid (FFA) content was used as the principal indicator of frying oil quality in industry. It was found that the changes in FFA level of the three different oils during frying had increased with the increasing of frying time (Figure 5). The FFA value of PS increased gradually from $0.05 \%$ (before frying) to $1.19 \%$ after the $5^{\text {th }}$ day of frying.

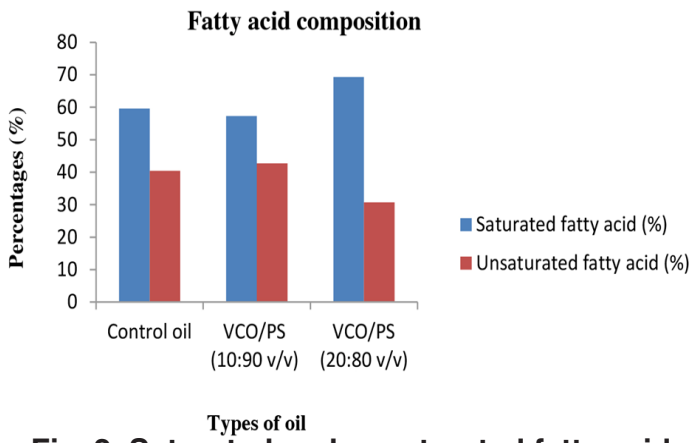

Fig. 2: Saturated and unsaturated fatty acid composition of the blends before frying

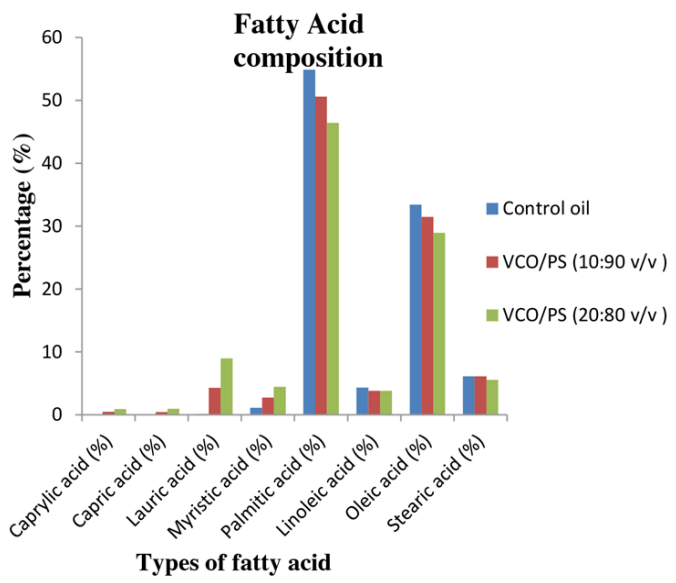

Fig. 4: Fatty acid composition (FAC) in blended shortenings after the $5^{\text {th }}$ day of frying 
When blending, the VCO: PS (10:90) blend, the FFA values were increased from $0.05 \%$ to $1.20 \%$ while in VCO: PS (20:80) blend, it increased from $0.05 \%$ to $1.15 \%$. It was also found that, the VCO: PS (20:80) blend has the lowest FFA content whereas the highest percentage of FFA was in VCO: PS (10:90) blend (Figure 4). According to Innawong 20 , the increase in FFA value might be due to the hydrolysis process in the presence of water from food and high temperature of frying. The result of VCO: PS (20:80) blend was the lowest content of FFA due the higher saturated fatty acid (SFA) content as SFA will reduce the rate of hydrolysis during the frying. However, FFA values in all three different blends are still far below the maximum discard point of $2-2.5 \%{ }^{21}$.

\section{Total Polymer Materials (TPM)}

Based on the result obtained, the TPMs showed a different trend in PS and VCO:PS blends. After frying for five consecutive days, the polymer formation was higher in PS than VCO:PS blends

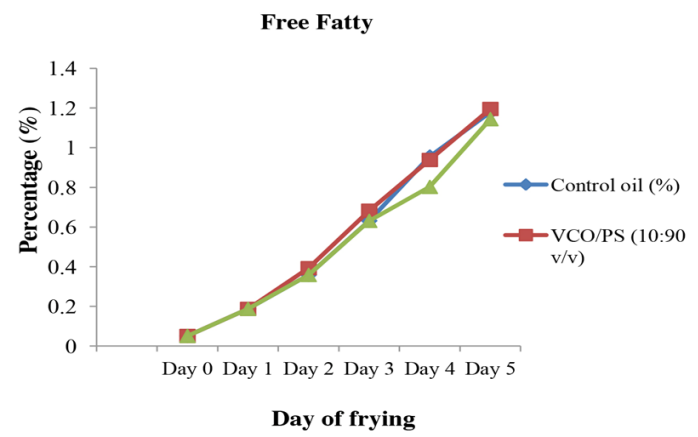

Fig. 5: Free fatty acid (FFA) values of blended shortening during frying

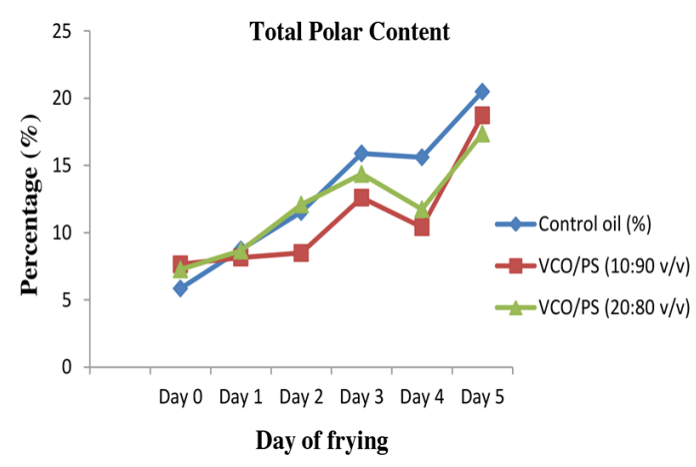

Fig. 7: The total polar (TPC) contents of blended shortenings during frying
(Figure 6). The TPM in VCO: PS (20:80) blend was the lowest $(1.40 \%)$ compared to TPM values in VCO: PS (10:90) blend (2.20\%) and in PS (3.02\%). The polymer compounds are formed as a result of oxidative and thermal reaction ${ }^{22}$ which lead to the formation of volatile and non volatile decomposition product due to oil degradation. Paradis and Newar ${ }^{23}$ also confirmed that the higher content of saturated fatty acids are reliable indicators of oil quality. The VCO: PS (20:80) blend has the lowest TPM throughout frying due to the high in saturated fatty acids and low in unsaturated fatty acid contents. On the $5^{\text {th }}$ day of frying, the percentage of saturated fatty acid in VCO: PS (20:80) was $67.3 \%$ compared to in VCO: PS (10:90) blend (64.7\%) and in PS $(62.3 \%)$. From this result, it can be concluded that both VCO:PS blends have lower TPMs than PS since these blends are able to resist the oxidation as they contain higher amount of saturated fatty acid and lauric acid compared to the control oil (PS). However, in the present study, all frying blends exhibited the

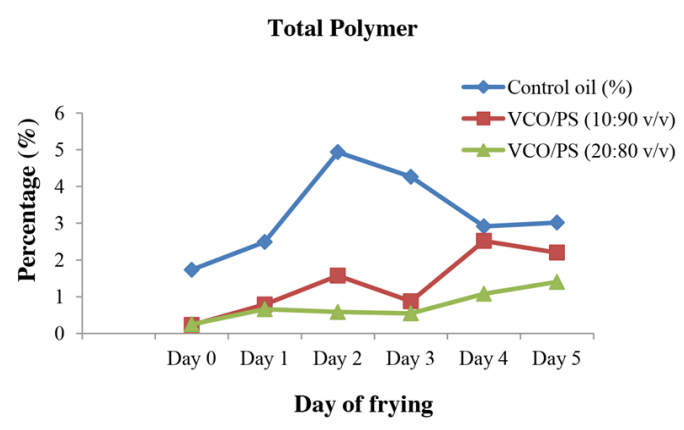

Fig. 6: The total polymer material (TPM) contents of blended shortenings during frying

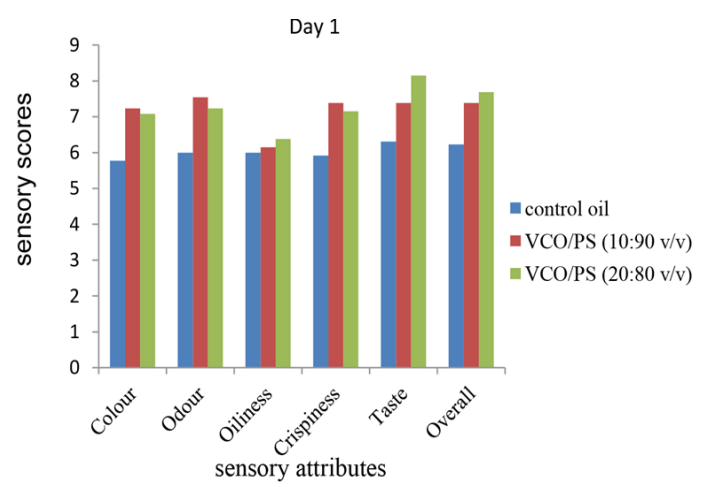

Fig. 8: Mean sensory score of fried chickens in control oil (PS), VCO:PS $(10: 90)$ and VCO:PS (20:80) of Day 1 
TPM values were still far below the discard point of $10-16 \%{ }^{21}$.

\section{Total Polar Compound (TPC)}

The results of the TPC contents in three different blends during frying process were shown in (Figure 7). The percentage of the TPCs in all blends were changed against frying time. VCO: PS (20:80) blend tended to have the lowest value of TPC while PS has the greatest value. The TPC in VCO: PS $(20: 80)$ blend increased to $17.4 \%$ after the $5^{\text {th }}$ day of frying. While in the VCO: PS (10:90) blend and PS, the TPC values were increased to $18.7 \%$ and $20.5 \%$ respectively. Nimitpornsuko ${ }^{24}$ reported that frying oil with high content of unsaturated fatty acid is more susceptible to oxidative degradation. Since total polar compounds (TPCs) are product of oil degradation, the high percentage of TPC in PS indicated that it was less stable during frying than VCO:PS blends. It was probably due to low content of saturated fatty acid compared to oil blends as reported earlier ${ }^{25}$. However, the TPC values in all blends were below

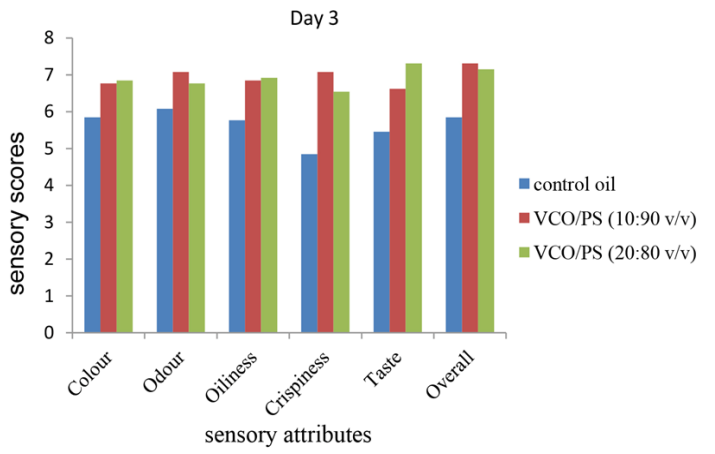

Fig. 9: Mean sensory score of fried chickens in control oil (PS), VCO:PS (10:90) and VCO:PS $(20: 80)$ of Day 3

significantly different $(p<0.05)$ from fried chickens in palm based solid shortening.

\section{CONCLUSION}

This study found that virgin coconut oil (VCO) can improve the frying performance of palm based solid shortening (PS) during deep frying. The physicochemical properties of VCO:PS blends were better than palm solid shortening (PS) alone. In term of oil uptake, the chickens fried in VCO:PS blends absorbed less oil than in palm shortening (PS). Moreover, in term of sensory evaluation, the addition the descard limit of $24 \%{ }^{21}$, thus the used blends were still suitable for human consumption.

\section{Sensoray Evaluation}

From the sensory mean scores on the Day 1, there were significant differences ( $p<0.05$ ) in terms of colour, odour, crispiness, taste and overall scores in all samples (Figure 8). In Day 3, the samples indicated that there were no significant difference in colour, odour and oiliness, while there was a significant difference $(p<0.05)$ in crispiness, taste and overall scores in all samples (Figure 9). On day 5 , there were no significant difference in colour, odour and crispiness while there was a significant difference $(p<0.05)$ in oiliness, taste and overall scores in all samples (Figure 10). Throughout this study, the addition of virgin coconut oil had affected the consumer perception on the fried chicken on oiliness, taste and overall score of fried chickens, although it did not contribute to the colour, odour and crispiness. The sensory data generally showed that fried chickens in virgin coconut oil were of

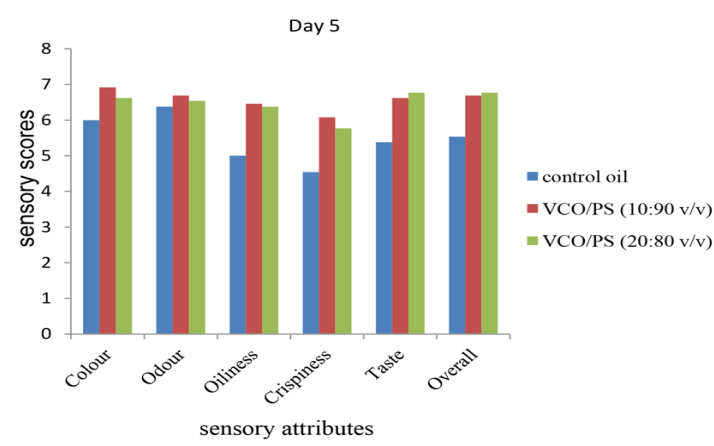

Fig. 10: Mean sensory score of fried chickens in control oil (PS), VCO:PS $(10: 90)$ and VCO:PS (20:80) of Day 5

of virgin coconut oil to palm based solid shortening showed significant differences on the oiliness, taste and overall acceptability of fried chicken after repeated frying. In overall, it can be concluded that the addition of a small proportion of VCO in palm based solid shortening gave a major improvement in frying stability of oil.

\section{ACKNOWLEDGEMENTS}

Thanks to the International Islamic University Malaysia (IIUM) and the Dean, Kulliyyah of Science for his permission to carry out this research. 


\section{REFERENCES}

1. Flegal, K. M.; Carroll, M. D.; Ogden, C. L.; Johnson, C. L. (2002). JAMA 2002,288, 1723-1727 doi:10.1001/jama.288.14.1723. PMID 12365955. http://jama.ama-assn.org/ cgi/content/full/288/14/1723.

2. Hill, J. O.; Wyatt, H. Obesity Res.2002, 10, 124S-130S doi: 10.1038/oby.2002.205; http:// dx.doi.org/10.1038/oby.2002.205

3. Haslam, D; James, W. P. (2005). Obesity. The Lancet 2005, 366, 1197-1209 doi:10.1016/ S0140- 6736(05)67483-1; http://dx.doi. org/10.1016/S0140-6736(05)67483-1; PMID 16198769.

4. Bray, G. A. J. Clin. Endocrinol. Metab.2004,89, 2583-2589 doi:10.1210/jc.2004-0535. http:// dx.doi.org/10.1210/jc.2004-0535; PMID 15181027.

5. Lew, E. A.; Garfinkel, L. (1979). Variations in mortality by weight among 750,000 men and women. J. Chron. Dis.1979,32, 563-576. http://www.ncbi.nlm.nih.gov/pubmed/468958 ?dopt=Abstract\&holding=npg.

6. Razali, I.; Badri, M.Palm Oil Dev. 2003,38, 1215 http ://palmoilis.mpob.gov. my/publications/ pod3 8-p11.pdf

7. Omar, M. N.; Nor-Nazuha, M. N.; Nor-Dalilah, M. N.; Sahri, M. M. Pak.J.Biol. Sci. 2010,13, 298-302 doi: 10.3923/pjbs.2010.298.302; http://dx.doi.org/10.3923/pjbs.2010.298.302

8. Farhani, M. R. Frying performance of virgin coconut oil and palm olein blends, FYP thesis, International Islamic University Malaysia, Kuantan, (2010)

9. Sundram, K. Palm Oil Dev.2009,42, 24-26.

10. Nour, A. H.; Mohammed, F. S.; Yunus, R. M.; Arman, A. Inter. J. Chem. Technol. 2009, 1, 59-64 doi: 10.3923/ijct.2009.59.64; http:// dx.doi.org/10.3923/ijct.2009.59.64

11. Nevin, K. G.; Rajamohan, T. Clin. Biochem.2004, 37, 830-835 doi:10.1016/j. clinbiochem.2004.04.010, http://dx.doi. org/10.1016/j.clinbiochem.2004.04.010

12. Ghazali, H. M.; Tan, A.; Abdulkarim, S. M.; Dzulkifly, M. H. J.FdAgric. Environ. 2009, 7, 2327 http://www.isfae.org/scientficjournal/2009/ issue3/pdf/food/4.pdf

13. Nor-Hazwani, M. H. Oil absorption and deep- frying performance using virgin coconut oil and palm based solid shortening, FYP Thesis, International Islamic University Malaysia, Kuantan, 2011

14. Dobarganes, C.M.; Velasco, J.; Dieffenbacher, A. Pure Applied Chem.2000,72, 1563-1575 doi:10.1351/pac200072081563; http:// dx.doi.org/10.1351/pac200072081563ht tp://195.37.231.82/publications/pac/pdf/2000/ pdf/7208x1563.pdf

15. Dobarganes, C.M.; Marquez-Ruiz, G. In Deep Frying: Chemistry, Nutrition and Practical Applications, AOCS Press, Champaign, 2007.

16. MPOB Test Method. A compendium of tests on palm oil products, palm kernel products, fatty acids, food-related products and others. Malaysian Palm oil Board, Bangi, Malaysia, 2004.

17. AOCS Official Method Ca 5a-40. Free fatty acid. American Oil Chemists' Society, Urbana, IL., 1997.

18. Kita, A.; Lisinska, G. (2005). J. Sci. Fd. Agric. 2005,85, 2600-2604 doi: 10.1002/jsfa.2319, http://dx.doi.org/10.1002/jsfa.2319

19. Marina, A. M.; Che Man, Y. B.; Nazimah, S. A.H.; Amin, I. Int. J. Fd. Sci. Nutr. 2009,60, 114-123 PubMed: 19115123

20. Innawong, B. Improving fried product and frying oil quality using nitrogen gas in a pressure frying system. PhD thesis, Virginia Polytechnic Institute and State University, Blacksburg, (2001)

21. Ismail, R.; Minal, J.; Sudin, N. A. Palm Oil Dev.2003,38, 1-4. http://mpob.gov.my/en/ component/content/article/181-publicationsback-issues/798-palm-oil-developmentsissn-0127-3329

22. Paradis, A. J.; Nawar, W. W. J. Am. Oil Chem. Soc.1981,58, 635-638 doi: 10.1007/ BF02672382; http://dx.doi.org/10.1007/ BF02672382

23. Tompkins C.; Perkins, E.G. J. Am. Oil Chem. Soc.2000, 77, 223-229 doi: 10.1007/s11746000-0036-2; http://dx.doi.org/10.1007/ s11746-000-0036- 2

24. Nimitpornsuko, N. Changes in Physical and Chemical Qualities of Used Frying Oils for 
Banana Slices. Mohidol University Publisher, Bangkok, Thailand, (2008)

25. Sulieman, A. E. M.; El-Makhzangy, A.; Ramadan, M. F. J. Fd. Lipids 2006,13, 259-
276 doi:10.1111/j.1745-4522.2006.00050.x; http://dx.doi.org/10.1111/j.17454522.2006.00050.x 